\title{
Differentiation of UC-MSCs into hepatocyte-like cells in partially hepatectomized model rats
}

\author{
ZHENG CHEN, QIAOTING KUANG, XUE-JUN LAO, JIE YANG, WEIDONG HUANG and DONG ZHOU \\ Department of General Surgery, Xiangyang Hospital Affiliated to Hubei University of Medicine, \\ Xiangyang, Hubei 441000, P.R. China
}

Received February 18, 2016; Accepted July 25, 2016

DOI: $10.3892 /$ etm.2016.3543

\begin{abstract}
The aim of the study was to investigate the possibility of human umbilical cord mesenchymal stem cells (UC-MSCs) surviving and differentiating into hepatocyte-like cells in partially hepatectomized model rats. MSCs were isolated from human umbilical cord and cultured with collagenase digestion. Cell surface markers were detected and fifth generation UC-MSCs were labeled with PKH26. The partially hepatectomized model rats were injected with the labeled human umbilical cord MSCs and transplanted through the portal vein. The survival of the labeled cells, in differentiation conditions and the expression of hepatic marker albumin were observed at post-transplantation 1,2 and 3 weeks under a fluorescence microscope. It was found that the human umbilical cord MSCs could be cultured and amplified in vitro. Following transplantation to the partially hepatectomized liver of the model rat, the cells survived and expresses the hepatic marker albumin in vivo. After being labeled with PKH26, the cells were visualized as red fluorescence under a fluorescence microscope. In the frozen sections of the liver, the marked cells scattered around and most of them expressed albumin with green fluorescence under the fluorescence microscope. In conclusion, the transplanted human umbilical cord MSCs survived and differentiated into hepatocyte-like cells. The human umbilical cord MSCs may therefore be a main source of hepatocytes in transplantation.
\end{abstract}

\section{Introduction}

Mesenchymal stem cells (MSCs) originate from mesoderm and can differentiate into three germ layers. MSCs are widely used in cell engineering research. They are mainly derived from bone marrow, and aer also found in fat in limited numbers $(1,2)$. Factors such as susceptibility to viral infections and strong immunogenicity limits their clinical applications (3).

Correspondence to: Dr Jie Yang, Department of General Surgery, Xiangyang Hospital Affiliated to Hubei University of Medicine, 15 Jiefang Road, Xiangyang, Hubei 441000, P.R. China

E-mail: 1692710644@qq.com

Key words: mesenchymal stem cells, hepatocyte, stem cells transplantation, in vivo, differentiation
Recent findings have shown that MSCs from human umbilical cord have advantages such as large numbers, strong proliferation and differentiation capacity and low immunogenicity (4) compared to MSCs in the bone marrow. MSCs originating from bone marrow differentiated into hepatocytes in partially hepatectomized models (5). However, there are few reports on whether human umbilical cord MSCs are capable of surviving and differentiating into hepatocyte-like cells in partially hepatectomized model rats.

In the present study, labeled human umbilical cord MSCs were transplanted into partially hepatectomized model rats, and the possibility of differentiating into hepatocytes in this regeneration environment of liver cells was examined.

\section{Materials and methods}

Main reagents. Reagents used were: Dulbecco's modified Eagle's medium (DMEM/F12; HyClone, Logan,UT,USA), fetal bovine serum (FBS; Gibco, Grand Island, NY, USA), trypsin (Solarbio, Beijing, China), PKH26 staining solution (Sigma, St. Louis, MO, USA), mouse anti-human albumin antibody (Dako, Glostrup, Denmark), and FITC-labeled double-antibody (Beijing Zhongshan Golden Bridge Biotechnology Co., Ltd., Beijing, China).

After written informed consent was obtained from the family or relatives of the patient, umbilical cord was collected from full term cesarean section under strict sterile conditions.

Experimental animals. Clean 6-week-old Sprague-Dawley female rats were purchased from the Guangdong Experimental Animal Center [license no. SCXK (Guangdong) 2008-0002]. Approval for the study and use of the animals was obtained from the ethics committee of Xiangyang Hospital (Hubei, China).

Isolation, cultivation and proliferation of umbilical cord MSCs (UC-MSCs). Four to six centimeters of healthy fetal umbilical cord was collected under strict sterile conditions and washed with PBS. The residual blood of the umbilical vein and the umbilical artery were rinsed off and the outer membrane and vascular tissues were removed. The umbilical cord was dissected into approximately $1 \mathrm{~mm}^{3}$ tissue blocks, and placed into the collagenase, the mass fraction of which was $0.1 \%$. After 20 -h digestion at $37^{\circ} \mathrm{C}$, the solution was filtered through a 100 mesh strainer and the filtrate with cells was collected. 
Subsequently, the filtrate was centrifuged at $290 \mathrm{x}$ g at $37^{\circ} \mathrm{C}$ for $10 \mathrm{~min}$ at room temperature, and the supernatant was discarded to retain the precipitate. The precipitate was washed twice with PBS and inoculated with a cell density of $1 \times 10^{6} \%$ $\mathrm{ml}$ in a T-75 plastic culture flask and cultivated in DMEM/ F12 culture medium [comprising 10\% (v/v) FBS, $100 \mu / \mathrm{ml}$ penicillin, $100 \mu / \mathrm{ml}$ streptomycin] under a saturated humid environment at $37^{\circ} \mathrm{C}$. After 4-5 days, $5 \%(\mathrm{v} / \mathrm{v})$ of the solution was initially altered. The non-adherent cells were discarded and the medium was changed every 2-3 days. When the cell fusion was up to $80 \%$, the cells were digested with $0.25 \%(\mathrm{v} / \mathrm{v})$ of trypsin for $5 \mathrm{~min}$. The cells at ratio of cell passage was 1:2 and were continued to cell expansion and cultivation.

Cell phenotype using flow cytometry (FCM). Fifth generation of cells with stable proliferation were taken, and digested with $0.25 \%(\mathrm{v} / \mathrm{v})$ of trypsin. PBS solution was used to wash cells twice and each tube was adjusted to $0.1 \mathrm{ml}$ with the cell density of $1 \times 10^{6} / \mathrm{ml}$. Subsequently, mouse anti-human monoclonal antibodies, CD29-FITC (cat. no.: 032041-M35 with a dilution of 1:200), CD13-FITC (cat. no.: 032041-M19 with a dilution of 1:500), CD44-FITC (cat. no.: 032041-M48 with a dilution of 1:500), CD31-FITC (cat. no.: 032041-M37 with a dilution of 1:500), CD106-FITC (cat. no.: 032041-M78 with a dilution of 1:200), CD45-FITC (cat. no.: 032041-M52 with a dilution of 1:500) were added and incubated at $4^{\circ} \mathrm{C}$. The cells were washed with PBS once, and detected using Cytomics ${ }^{\mathrm{TM}}$ FC500 FCM (Beckman Coulter, Brea, CA, USA), followed by analysis with Cytometer 1.0 software (Frederick, MD, USA).

Fluorescence-labeled UC-MSCs in vitro. Cells were digested into single cell suspension. Cells $\left(1 \times 10^{7}\right)$ were centrifuged at $500 \mathrm{rpm}$ for $5 \mathrm{~min}$ to form a loose cell mass. The supernatant was discarded and the rest was added into $1 \mathrm{ml}$ dilution $\mathrm{C}$ to resuspend the cells. Subsequently, the PKH26 dye solution that was diluted by dilution $\mathrm{C}$ was added to make a final concentration of $2 \mathrm{mmol} / \mathrm{l}$. The cells were mixed with the dye solution, and incubated at $25^{\circ} \mathrm{C}$ for $5 \mathrm{~min}$. The same volume of serum was then added to terminate the reaction. The same volume of serum-containing culture solution was added to dilute the solution, and centrifuged for $10 \mathrm{~min}$ at $100 \mathrm{x} \mathrm{g}$, followed by washing 3 times. Then, $2 \times 10^{6} / \mathrm{ml}$ of cell suspension was formed with culture medium and cell staining was observed under a fluorescence microscope (Thermofisher, Beijing, China).

Development of the partially hepatectomized rat model. Six-week-old Sprague-Dawley rats were anesthetized by injecting $2 \%(35 \mathrm{mg} / \mathrm{kg})$ pentobarbital in the abdominal cavity. The rats were placed on a sterile operating table in a supine position, followed by disinfection of the abdomen skin with alcohol prior to dissecting the abdominal cavity. Subsequently, the thorax of the rat was gently squeezed to visualize the liver clearly and the liver lobe was double ligated at the hepatic pedicle of the diaphragmatic lobe of the liver. The hepatic vein was cut and the portal vein was separated to inject slowly the $0.5 \mathrm{ml}$ cell suspension (approximately $1 \times 10^{6}$ cells), already marked with the staining solution, $\mathrm{PKH} 26$, by using a $1 \mathrm{ml}$ syringe. The bleeding was quickly stopped by pressing, and spraying a small amount of penicillin solution into the abdominal cavity, after which it was sutured. Once the rat recovered from the anaesthesia, regular feeding was continued.

Observation of the sliced liver and staining using albumin immunofluorescence. After cell transplantation, parts of the liver were dissected during the subsequent three weeks. The sections (5 $\mu \mathrm{m}$ ) were frozen, and observed by fluorescence microscopy to confirm whether there were any red-labeled cells in the liver. Subsequently, the anti-albumin antibody (cat. no.: K08531 with a dilution of 1:100) was added for incubation at $4^{\circ} \mathrm{C}$ overnight. The following day, tissues were warmed briefly at $37^{\circ} \mathrm{C}$ for $30 \mathrm{~min}$, followed by washing twice with PBS. FITC-labeled secondary antibodies were added at $37^{\circ} \mathrm{C}$ for $1 \mathrm{~h}$, and washed 3 times with PBS. The sections were mounted with glycerol and observed immediately under a fluorescence microscope.

\section{Results}

Isolation, cultivation and morphologic observation of human umbilical cord MSCs. The single cells obtained by collagenase digestion began to adhere within $24 \mathrm{~h}$ in primary culture. After 5 days, the majority of cells presented the phenomenon of adherence, and most of the cells were of diamond shape (Fig. 1A). Each 2 days, the cells were passaged once, and thereafter the cells proliferated rapidly and the number of passages went up to 20 generations. After the passaging, the cells were of high purity, uniform shape, and grew in a spiral shape (Fig. 1B).

Cell phenotype analysis of human umbilical cord MSCs. Using FCM, the cells showed stromal markers and adhesion molecules CD29, CD44, CD13, and indicated a low expression of CD106. By contrast, the cells did not show any sign of endothelial cell marker CD31 and hematopoietic stem cell flag CD45 (Fig. 2), indicating that these cells had features of stem cells, which was in line with the requirements of this experiment.

Observation of in vitro PKH26 staining of the human umbilical cord MSCs. After staining, the marker was distinguished for $22 \mathrm{~h}$ (Fig. 3A), at $45 \mathrm{~h}$ (Fig. 3B) and observed in the suspended state (Fig. 3C),. The red fluorescence marked in the human umbilical cord MSCs was observed under fluorescence microscopy. No significant difference was observed in cell growth, morphology and function after passaging between the labeled and unlabeled cells.

Positioning of MSCs in the liver and determining the expression of cell albumin by utilizing immunofluorescence. Rats were sacrificed in the first, second, and third week, after which the abdominal cavity was opened to observe the liver. There were clear and broad adhesions between the liver and surrounding tissues, the surface of the liver was uneven, and at the ligature where part of the liver was cut, the tissue was firm or hard. The rat liver was dissected to make frozen sections and the position of the labeled cells in liver was observed under the fluorescence microscope. The tagged red fluorescence cells were scattered in the liver and some were embedded in the liver panel (Fig. 4A-C). Due to cell differentiation, the red fluorescence gradually faded and after immunofluorescence staining, labeled cells with albumin staining were detected as positive, and excited green fluorescence (Fig. 5A-C), indicating 

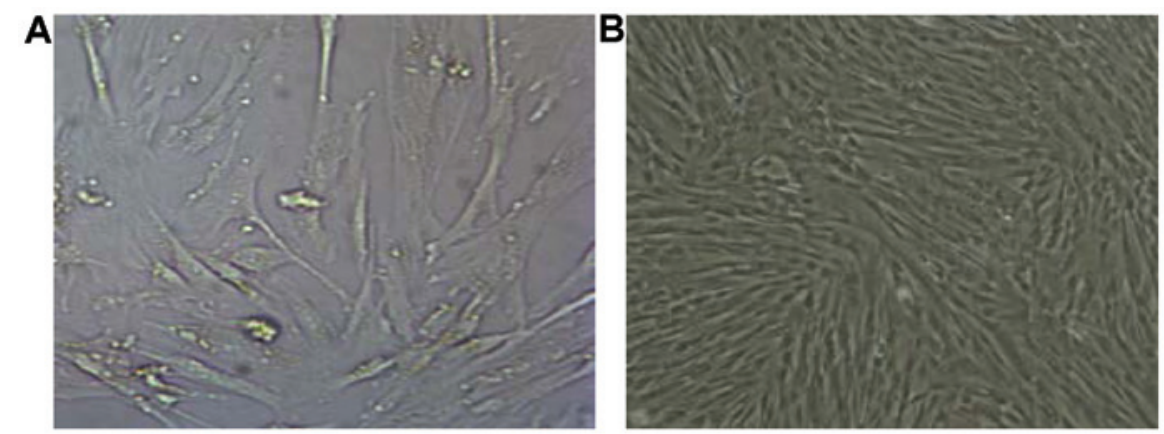

Figure 1. (A) MSCs of the second generation (x50). (B) MSCs of the fifth generation (x4). MSCs, mesenchymal stem cells.
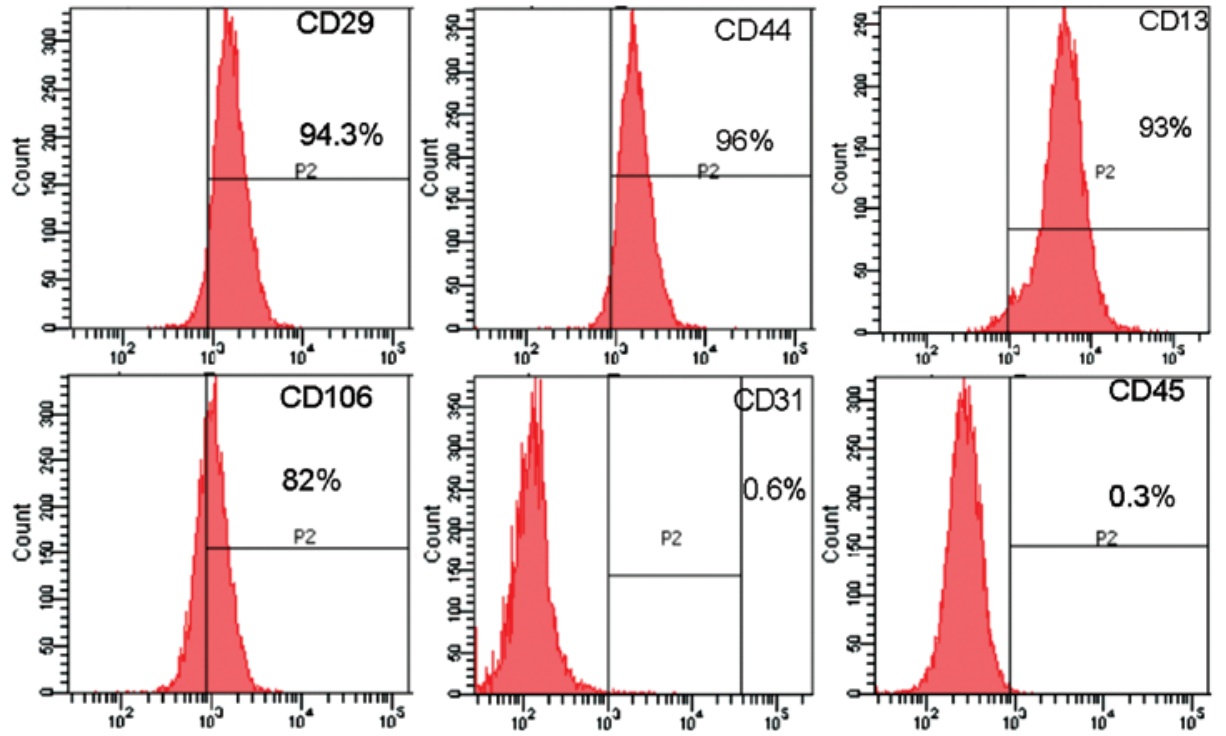

Figure 2. The cell phenotype of the human UC-MSCs. UC-MSCs, umbilical cord mesenchymal stem cells.
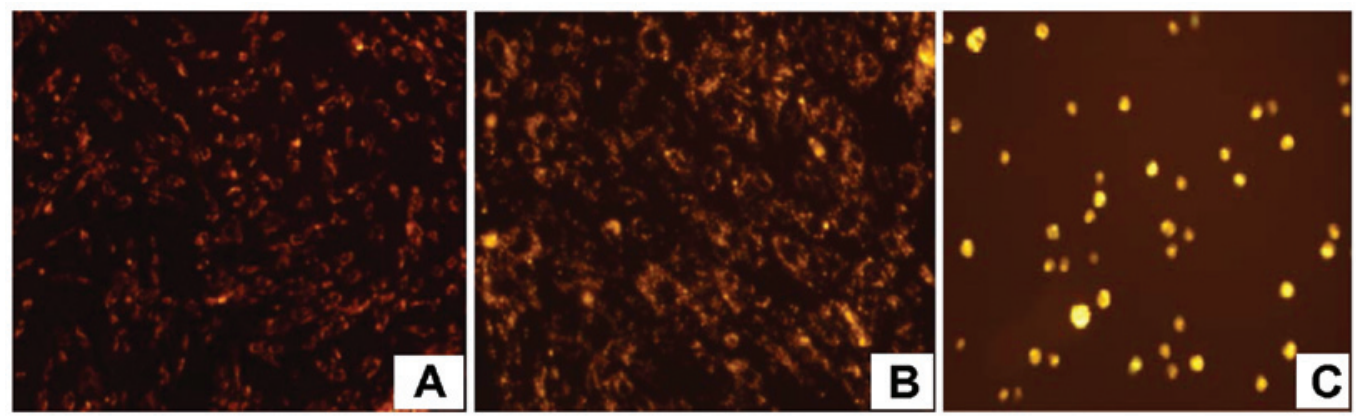

Figure 3. (A) MSCs (x100) after dyeing 22 h; (B) MSCs (x100) after dyeing 45 h; (C) MSCs (x100) in suspension. MSCs, mesenchymal stem cells.
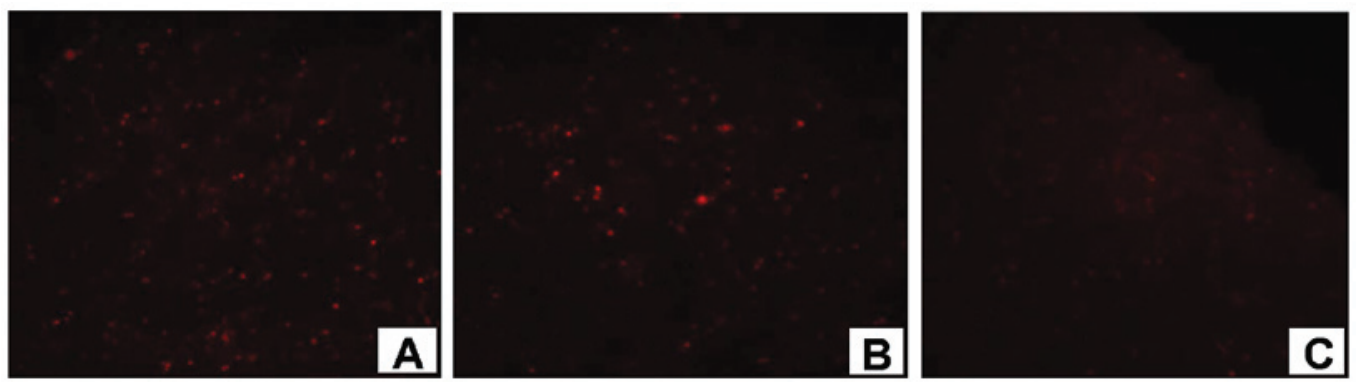

Figure 4. (A) MSCs (x100) in the first week; (B) MSCs (x100) in the second week; (C) MSCs (x100) in the third week. MSCs, mesenchymal stem cells. 

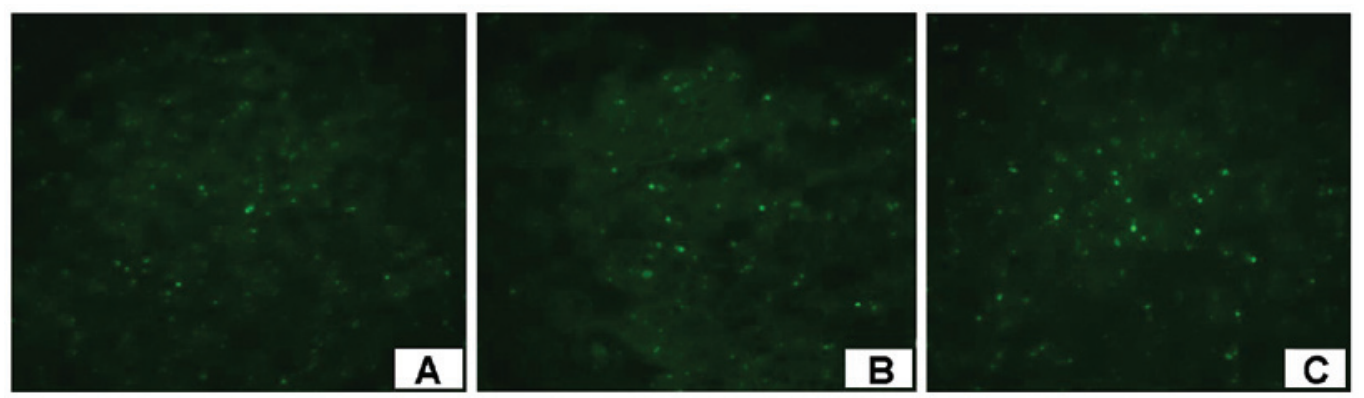

Figure 5. (A) Expression of albumin in MSCs (x100) in the first week; (B) the expression of albumin in MSCs (x100) in the second week; (C) the expression of albumin in MSCs (x100) in the third week. MSCs, mesenchymal stem cells.
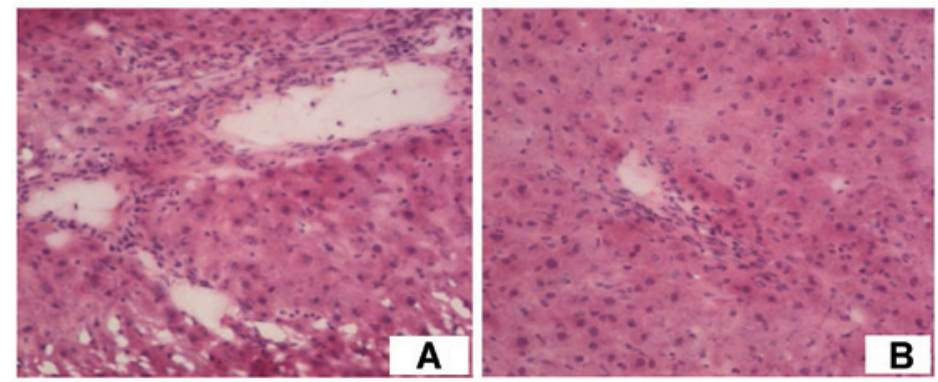

Figure 6. (A) liver of the model rats in the second week (x40); (B) liver of model rats in the third week (x40).

that the human umbilical cord produces white protein. In addition, after H\&E staining there were a large number of cells aggregating around the hepatic sinusoid, and hyperplasia was relatively active (Fig. 6A and $\mathrm{B}$ ).

\section{Discussion}

As a temporary organ, the umbilical cord is a relatively simple structure, mainly rich in Wharton's jelly of collagen as well as vascular and mesenchymal elements (6). Many experiments have shown that with suitable induction in vitro, human umbilical cord MSCs may differentiate into mesodermal cells, such as osteoblasts, muscle cells, or ectodermal and endodermal liver cells, such as neural glial cells $(7,8)$. Umbilical cord is a rich source of stem cells that are easier to culture and proliferate, giving UC-MSCs great clinical value.

In the present study, using collagenase digestion, we successfully isolated and cultured human umbilical cord MSCs. To provide an in-depth understanding of how the transplanted cells in vivo repaired damaged tissues, many cell labeling methods have been used (9). Achievement of an appropriate, effective, and practical cell labeling technique, remains a challenge. PKH26 is a lipophilic fluorescent dye that irreversibly binds to the cell membrane $(10,11)$. It is excited in red fluorescence and in the exposure of 527-nm wavelength exerted little influence on cell viability and proliferation ability. It is therefore a relatively good tracing marker in vivo. The cell fluorescence labeled by PKH26 may be kept inside the body at least for one month $(12,13)$. With division of cells, the fluorescent dye was almost equally distributed into two daughter cells and the fluorescence intensity of the daughter cells also decreased. As the cells continued to differentiate, the red fluorescence gradually faded (14-17). Several studies have shown that the MSCs of bone marrow or fat of a model rat successfully differentiated into liver cells in other partially hepatectomized model rats. The method in MSCs was induced to differentiate them into hepatic cells in vivo avoiding the difficulties and limitations in vitro and making MSCs directly involved in the liver injury repair (18-20). As this experiment was heterogeneous allograft and the microenvironment was different in vivo there was a high chance of immune rejection. FCM detected that surface markers of human umbilical cord MSCs were the same as the fetal lung tissue-derived MSCs (21-23), but did not express HLA-DR, which was the main factor to cause the immune response, suggesting that the relative immunogenicity of human umbilical cord MSCs was relatively weak and was appropriate to be transplanted between different individuals (24-27). At the same time, after portal vein transplantation, the cells directly reached the liver, which provided a better microenvironment for cell growth. Therefore, it is feasible to observe the positioning and differentiation of cells in the liver in an improved manner. Partial liver resection is the optimal model of liver regeneration. Liver resection caused an increase in hepatocyte growth signals, such as metabolic nutritional factors and neurohormones, providing a good microenvironment for the regeneration of liver cells (28-31). The growth signals in the blood also induced the stem cells to express hepatocyte markers (32). In this experiment, a heterogeneous stem cell transplantation model was established on the basis of the experimental model of partial hepatectomy. This was similar to the clinical experimental model, as the donor cells were screened and prepared in advance and were ready for immediate use. Transplanted cells were successfully implanted and survived for a long time in rats, indicating that this method is safe, reliable, and there were no significant hyperacute or acute rejection of the transplantation. Liver after partial hepatectomy regenerated significantly within 2 weeks, 
and finished regeneration within three months. In this process, the residual liver cells regenerated and died simultaneously (11), thus in this study, the liver of the model rat was cut at the first, second and third week and frozen. Under a fluorescent microscope, it was evident that stem cells were scattered in the liver with intact cell structures. Part of the liver cells were embedded in the hepatic plate with liver cell morphology, and expression of albumin was detected with anti-human albumin antibody. Along with cell differentiation, the red fluorescence faded away, while the green fluorescence, which represented the albumin expression was enhanced, indicating that after transplantation the human umbilical cord MSCs were able to differentiate into hepatocytes in vivo, and participate in the regeneration of liver cells. We used anti-human albumin antibody, despite taking the differentiation potential of human umbilical cord MSCs into account, to exclude the interference of albumin generated by the liver cells of rats and prevent the generation of false positives.

In conclusion, human umbilical cord MSCs were implanted into the model rats via portal vein transplantation. This confirmed that the human umbilical cord MSCs differentiated into hepatocytes in the allograft and liver regeneration environment and there was no significant adverse reactions without the use of immunosuppressants. By combining the experience of clinical practice, the umbilical cord MSCs can become a promising cell source for bioartificial liver system and liver cell transplantation and bring hope to patients with advanced liver cancer. However, this is only an experimental animal study, thus, it is difficult to assess correctly the long-term treatment effect, and there remains a gap between the experimental and clinical application, which needs further study.

\section{Acknowledgements}

The study was funded by the Science and Technology funded projects of Guangdong Province (grant no. 2010B031600248).

\section{References}

1. Mezey E and Chandross KJ: Bone marrow: a possible altern ative source of cells in the adult nervous system. Eur J Pharmacol 4 297-302, 2000

2. Fukuda K and Preck D: Reprogramming of bone marrow mesenchymal stem cells into cardiomyocytes. C R Biol 325: 1027-1038, 2002.

3. Woodbury D, Schwarz EJ, Prockop DJ and Black IB: Adult rat and human bone marrow stromal cells differentiate into neurons. J Neurosci Res 61: 364-370, 2000.

4. Yu SC, Xu YY, Li Y, Xu B, Sun Q, Li F and Zhang XG: Construction of tissue engineered skin with human amniotic mesenchymal stem cells and human amniotic epithelial cells. Eur Rev Med Pharmacol Sci 19: 4627-4635, 2015.

5. He J, Cai Y,Luo LM and Liu HB: Hypoxic adipose mesenchymal stem cells derived conditioned medium protects myocardial infarct in rat. Eur Rev Med Pharmacol Sci 19: 4397-4406, 2015

6. Forraz N and McGuckin CP: The umbilical cord: a rich and ethical stem cell source to advance regenerative medicine. Cell Prolif 44 Suppl 1: 60-69, 2011

7. Lu LL, Song YP, Wei XD, Fang BJ, Zhang YL and Li YF: Comparative characterization of mesenchymal stem cells from human umbilical cord tissue and bone marrow. J Exp Hematol 16: 140-146, 2008 (In Chinese).

8. Zhan YT, Wang Y, Wei L, Liu B, Chen HS, Cong X and Fei R: Differentiation of rat bone marrow stem cells in liver after partial hepatectomy. World J Gastroenterol 12: 5051-5054, 2006.

9. Blute JW, Douglas T, Witwer B, Zhang SC, Strable E, Lewis BK et al: Magnetodendrimers allow endosomal magnetic labeling the in vivo tracking of stem cells. Nat Biotechnol 19: 1141-1147, 2011.
10. Ji KH, Xiong J, Fan LX, Meng HK and Liu HQ: Rat marrow derived multipotent adult progenitor cells differentiate into skin epidermal cells in vivo. J Dermatol 36: 403-409, 2009.

11. Wallace PK and Muirhead KA: Cells tracking 2007: a proliferation of probes and applications. Immunol Invest 36: 527-561, 2007.

12. Haas J, Bauer P, Rolf A and Wree A: Immunocytochemical characterization PKH26 labelled an intracerebrally transplanted neonatal cells. Acta Histochem 102: 273-280, 2011.

13. Fox D, Kouris GJ, Blumofe KA, Heilizer TJ, Husak V and Greisler HP: Optimizing fluorescent labeling of endothelial cells for tracking during long term studies of autologous transplantation. J Surg Res 86: 9-16, 2015.

14. Weiss ML and Troyer DL: Stem cells in the umbilical cord. Stem Cell Rev 2: 155-162, 2006.

15. Li B, Tian XB, Hu RY, Xu FB and Zhao JM: Mechanism of BMP and TG2 in mesenchymal stem cell osteogenesis. Eur Rev Med Pharmacol Sci 19: 4214-4219, 2015.

16. Xue Z, Niu LY, An G, Guo YS, Lv SC and Ren XP: Expression of recombinant BMP-7 gene increased ossification activity in the rabbit bone mesenchymal stem cells. Eur Rev Med Pharmacol Sci 19: 3056-3062, 2015.

17. Hendrikx PJ, Martens CM, Hagenbeek A, Keij JF and Visser JW: Homing of fluorescently labeled murine hematopoietic stem cells. Exp Hematol 24: 129-140, 1996.

18. Oyagi S, Hirose M, Kojima M, Okuyama M, Kawase M, Nakamura T, Ohgushi $\mathrm{H}$ and Yagi K: Therapeutic effect of transplanting HGF-treated bone marrow mesenchymal cells into CCl4-injured rats. J Hepatol 44: 742-748, 2006.

19. Zhang Y, Tang CL, Chen WJ, Zhang Q and Wang SL: Dynamic compression combined with exogenous SOX-9 promotes chondrogenesis of adipose-derived mesenchymal stem cells in PLGA scaffold. Eur Rev Med Pharmacol Sci 19: 2671-2678, 2015.

20. Han YF, Sun TJ, Han YQ, Xu G, Liu J and Tao R: Clinical perspectives on mesenchymal stem cells promoting wound healing in diabetes mellitus patients by inducing autophagy. Eur Rev Med Pharmacol Sci 19: 2666-2670, 2015.

21. Javazon EH, Beggs KJ and Flake AW: Mesenchymal stem cells: paradoxes of passaging. Exp Hematol 32: 414-425, 2004.

22. Wang J, Lu Y, He DM and Zhang Y: Isolation, purification and identification of mesenchymal stem cells derived from human umbilical cord. J Jinan University 17: 367-372, 2009 (In Chinese).

23. Jia Z: Basic therapy and clinic of hepatopathy cells Beijing: People's Medical Publishing House, pp139-140, 2005.

24. Ma J, Duan FL, Yan FG, Li WX, Wang X, Chen XY, Gao TH, Zhu WL and Wang ZQ: Serum from partial hepatectomy rat and hepatocyte growth factor stimulate bone marrow cell expressing albumin and alpha fetoprotein. Zhonghua Gan Zang Bing Za Zhi 12: 410-413, 2004 (In Chinese).

25. Li JW and Wu X: Mesenchymal stem cells ameliorate LPS-induced acute lung injury through KGF promoting alveolar fluid clearance of alveolar type II cells. Eur Rev Med Pharmacol Sci 19: 2368-2378, 2015 .

26. Zhao YF, Luo YM, Xiong W, Ding W, Li YR, Zhao W, Zeng HZ, Gao HC and Wu XL: Mesenchymal stem cell-based FGF2 gene therapy for acute lung injury induced by lipopolysaccharide in mice. Eur Rev Med Pharmacol Sci 19: 857-865, 2015.

27. Garcea G and Maddern GJ: Liver failure after major hepatic resection. J Hepatobiliary Pancreat Surg 16: 145-155, 2009.

28. Sun TJ, Tao R, Han YQ, Xu G, Liu J and Han YF: Wnt3a promotes human umbilical cord mesenchymal stem cells to differentiate into epidermal-like cells. Eur Rev Med Pharmacol Sci 19: 86-91, 2015.

29. Roseti L, Serra M, Canella F, Munno C, Tosi A, Zuntini M, Pandolfi M, Sangiorgi L, Biso P, Pittalis MC, et al: In vitro gene and chromosome characterization of expanded bone marrow mesenchymal stem cells for musculo-skeletal applications. Eur Rev Med Pharmacol Sci 18: 3702-3711, 2014.

30. Xu Y, Sun DC, Wei ZT, Hong BF and Yang Y: Experimental study on transplantation of autologous minced muscle with human umbilical cord mesenchymal stem cells for urethral reconstruction. Eur Rev Med Pharmacol Sci 18: 3412-3419, 2014

31. Yao XL, Li L, He XL, Cui L, Kuang W and Tang M: Activation of $\beta$-catenin stimulated by mechanical strain and estrogen requires estrogen receptor in mesenchymal stem cells (MSCs). Eur Rey Med Pharmacol Sci 18: 3149-3155, 2014.

32. Zhu XW, Zuo JL, Liu YH, Zang R, Li YK, Wang X and Li JM: Osteogenesis of umbilical mesenchymal stem cells is enhanced in absence of DNA methyltransferase 3B (DNMT3B) through upregulating Runx2 expression. Eur Rev Med Pharmacol Sci 18: 3004-3009, 2014 